\title{
Effects of Conductive Electrode for Optimized Electrochemical Performances
}

\author{
Kue-Ho Kim ${ }^{1}$, Ki-Wook Sung ${ }^{2}$, and Hyo-Jin Ahn ${ }^{3 *}$ \\ 1,2,3 Seoul National University of Science and Technology \\ (Department of Materials Science and Engineering, Seoul, Korea)
}

\begin{abstract}
Recently, global challenges related to increasing energy consumption have attracted many researches regarding the energy conservation and efficiency increasement. This has accelerated a performance enhancement for various electronic devices used in daily life that based on electrochemical reaction (electrochemical capacitors, Li-ion batteries, electrochromic devices etc.). The electrochemical reaction refers to the induction of an oxidation-reduction reaction in a material which accompanied with electrons and ion movement. Their performances are determined by the electrochemical reaction rate and quantity which occurs at the interface of electrode and electrolyte. The typical electrochemical devices are composed of three parts including active material layer, electrolyte layer and conductive electrode layer. Among them, conductive electrode layer transfers electrons through an external circuit and the electrical conductivity of this layer can significantly affect to the electrochemical reaction rate. In this study, we demonstrated the effects of conductive electrode layer on electrochemical device by varying the electrical conductivity of them and investigated their performance enhancement mechanisms systematically.
\end{abstract}

Keywords: Conductive Electrode Layer; Electrical Conductivity; Electrochemical Devices; Energy Efficiency; Redox Reaction 